\title{
Avaliação da adaptação da corrente interferencial nas formas bipolar e tetrapolar em mulheres com lombalgia crônica não específica: ensaio clínico cruzado
}

\author{
Evaluation of interferential current adaptation in \\ bipolar and tetrapolar forms in women with chronic \\ non-specific low back pain: cross-over clinical trial
}

\author{
Katlen Ludke de Lima' ${ }^{1}$ \\ Priciane Tais Krampe ${ }^{2}$ \\ William Henrique Battisti ${ }^{3}$ (b) \\ Dérrick Patrick Artioli ${ }^{4}$ \\ Gladson Ricardo Flor Bertolini ${ }^{5}$ (c)
}

\begin{abstract}
${ }^{1-3}$ Universidade Estadual do Oeste do Paraná (Cascavel).Paraná, Brasil. katlenludke@hotmail.com, prici_tais@hotmail.com, william98battisti@gmail.com ${ }^{4}$ Centro Universitário Lusíada (Santos). São Paulo, Brasil. derricksantacasa@hotmail.com ${ }^{5}$ Autor para correspondência. Universidade Estadual do Oeste do Paraná (Cascavel). Paraná, Brasil. gladsonricardo@gmail.com
\end{abstract}

RESUMO | INTRODUÇÃO: A corrente interferencial é uma modalidade bastante utilizada, porém, apresenta como fator limitante a quantidade de acomodações, o que é comum em eletroestimulação. Apesar de possuir alguns recursos que visam reduzir a adaptação fisiológica, não é totalmente eficaz; neste sentido, pode haver diferenças devido à técnica de uso. Assim, o presente estudo pretendeu analisar se há diferenças na adaptação para as formas bipolar ou tetrapolar em indivíduos com dor lombar crônica não específica. MÉTODOS: Ensaio clínico cruzado, composto por 15 voluntárias com dor lombar crônica não específica, as quais receberam terapia bipolar ou tetrapolar em semanas subsequentes. Foi explicado sobre a adaptação à corrente e o que deveria avisar quando ocorresse, e, desta forma, foi computado o número de vezes em que o fenômeno ocorreu, o tempo necessário até ocorrer a primeira adaptação, a intensidade inicial da corrente utilizada e o quanto aumentou-se após a primeira adaptação. RESULTADOS: Para nenhuma das variáveis analisadas, foi observada diferença significativa entre as duas técnicas $(p>0,05)$. CONCLUSÃO: As técnicas analisadas não mostraram diferenças na adaptação em mulheres jovens com dor lombar não específica.

PALAVRAS-CHAVE: Dor lombar. Adaptação fisiológica. Terapia por estimulação elétrica.

\begin{abstract}
INTRODUCTION: The interferential current is a widely used modality; however, it presents as a limiting factor the amount of accommodation, which is common in electrostimulation. Despite having some resources that aim to reduce physiological adaptation, it is not fully effective in this sense, but there may be differences due to the technique of use. Thus, the present study aimed to analyze whether there are differences in the accommodation for bi- or tetrapolar forms in individuals with chronic nonspecific low back pain. METHODS: Crossover clinical study, consisting of 15 volunteers with chronic nonspecific low back pain, who received bipolar or tetrapolar therapy in subsequent weeks. They were explained about the current accommodation and that they should be told when it occurred, and in this way, the number of times that the phenomenon occurred, the time needed until the first accommodation occurred, the initial intensity of the current used, and how much it increased after the first accommodation were computed. RESULTS: None of the variables analyzed had a significant difference between the two techniques ( $p>0.05$ ). CONCLUSION: The techniques analyzed showed no differences in accommodation in young women with nonspecific low back pain.
\end{abstract}

KEYWORDS: Low back pain. Physiological adaptation. Electric stimulation therapy. 


\section{Introdução}

A dor lombar não específica afeta pessoas em todas as faixas etárias e caracteriza-se por não ter uma causa determinada. Gera alterações nas atividades cotidianas e pode ser iniciada por fatores físicos e/ou psicossociais, o que leva a perdas de dias de trabaIho e estudo, principalmente em sua forma crônica, a qual tem duração a partir de 3 meses. Apesar de seu curso clínico geralmente ser favorável, muitas vezes são necessárias terapias medicamentosas e não farmacológicas para seu tratamento.,1,2 Dentre as ferramentas utilizadas no tratamento fisioterapêutico, pode-se citar a corrente interferencial, a qual, apesar de controvérsias ${ }^{3,4}$, tem se mostrado efetiva em reduzir a dor lombar de forma isolada $a^{5,6}$ ou prévia a protocolos de exercícios. ${ }^{7}$

A corrente interferencial caracteriza-se por ser de média frequência (desta forma poder atingir tecidos profundos), em que duas correntes com pequena diferença em suas frequências, interferem uma na outra, gerando uma nova corrente modulada em baixa frequência. A principal finalidade de seu uso é a redução de quadros de dor. .99 Contudo, é muito comum, durante seu uso, a ocorrência da adaptação de nervos periféricos, necessitando que a intensidade da corrente seja aumentada para que os estímulos sejam adequados para seu objetivo. .9 A adaptação ocorre quando um estímulo é aplicado sobre um receptor neuronal, chega a um platô e os potenciais de ação tornam-se reduzidos em sua frequência, tal fenômeno é devido ao aumento da condutância do potássio e, posteriormente, à inativação de canais de sódio. $\frac{10}{}$

A corrente interferencial possui alguns recursos que visam reduzir a adaptação fisiológica ao estímulo constante, que são variações na frequência $(\Delta f)^{11} d a$ corrente modulada (AMF), entregues em determinados padrões de subida e descida ${ }^{12}$, contudo, em indivíduos saudáveis não têm alcançado seu objetivo, o que também tem sido observado quando alterando a frequência base da corrente ${ }^{13}$, porém, a forma bipolar mostrou-se acomodar mais rapidamente do que a tetrapolar em indivíduos saudáveis. ${ }^{14}$ Visto que a modalidade é utilizada geralmente em pessoas com dor, e que a adaptação gera necessidade de maiores cuidados diretos com o paciente, o presente estudo pretendeu analisar se há diferenças na adaptação para as formas bi ou tetrapolar em indivíduos com dor lombar crônica não específica.

\section{Materiais e métodos}

O presente estudo caracterizou-se como quase-experimental, de corte longitudinal, quantitativo de caráter cruzado, cego por parte do avaliador. O grupo amostral foi composto por 15 voluntárias do sexo feminino, selecionadas de forma direta e por conveniência.

No primeiro momento, foi explicado a cada voluntária sobre as intenções e procedimentos da pesquisa, bem como questionado sobre o interesse deste em participar da mesma. Sendo aceito o convite e presentes os critérios de inclusão e não os de não inclusão, foi assinado o termo de consentimento livre e esclarecido (TCLE), aprovado pelo comitê de ética em pesquisa (parecer número 3.325.098, CAAE 93672318.9.0000.0107).

Como critérios de inclusão, foram considerados: ser universitário, ter dor lombar persistente há pelo menos quatro meses, com idade entre 17 a 28 anos. Os critérios de não inclusão foram: dor lombar específi$\mathrm{ca}$, uso de drogas que afetassem o sistema nervoso central, prática de exercício físico, não concordância da participação do estudo, gravidez e doenças neuromusculoesqueléticas (além de dor lombar crônica).

As 15 voluntárias foram separadas em 2 subgrupos por sorteio (https://www.graphpad.com/quickcalcs/ randomize1/), para que de forma cruzada fosse alternado, com separação de 1 semana, a forma de terapia aplicada (bipolar ou tetrapolar) em todas as participantes. Os parâmetros utilizados foram: corrente base de $4 \mathrm{kHz}, \mathrm{AMF} 100 \mathrm{~Hz}$; $\Delta \mathrm{FO}$, com duração de 20 min, com diferença de aplicação do modo bipolar (eletrodos dispostos sobre processo espinhoso de L1 e L5 - fig. 1A), modo tetrapolar (aplicado lateralmente $3 \mathrm{~cm}$ dos processos espinhosos de $\mathrm{L} 1 \mathrm{e}$ L5 - fig. 1B), os eletrodos eram de borracha-silicone, com área de $8 \mathrm{~cm} 2$ cada. 

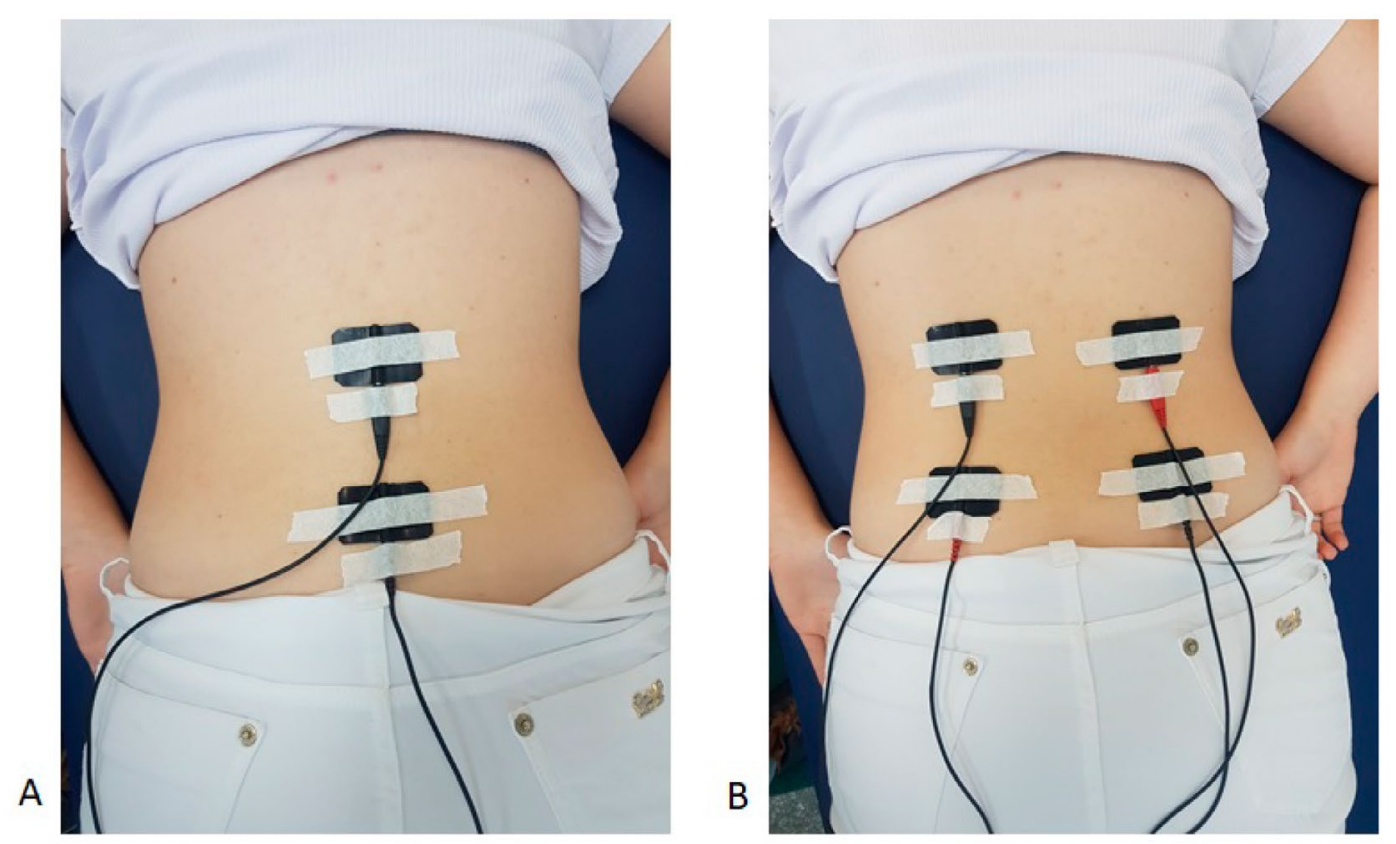

Foi explicado, prévio à aplicação da corrente, sobre a adaptação da corrente e que quando tal fenômeno ocorresse, elas deveriam solicitar que a intensidade fosse aumentada até que voltassem a sentir o mesmo estímulo inicial. As variáveis analisadas foram: a) o número total de acomodações; b) tempo necessário até ocorrer a primeira adaptação (em segundos); c) intensidade (em miliamperes) inicial; d) intensidade aumentada na primeira vez que a corrente acomodou. Sendo "a" e "b" desfechos primários e "c" e "d" secundários.

\section{Análise Estatística}

Os dados foram analisados pelo programa Biostat 5.0. A Avaliação de normalidade foi realizada pelo teste de Shapiro-Wilk, para os dados paramétricos foi utilizado teste t pareado e para os não paramétricos Wilcoxon, em todos os casos o nível de significância aceito foi de 5\%. Desta forma, a análise do total de acomodações e tempo para ocorrer a primeira adaptação foi apresentado em gráfico apresentando mediana e dispersão, e para a intensidade inicial e aumento da mesma o gráfico apresentou média e desvio-padrão. A amostra $(n=15)$ foi calculada com base em tamanho de efeito de 0,9, $\mathrm{a}=0,05$ e poder de $95 \%$ ( $G *$ Power 3.1.9.7).

\section{Resultados}

A amostra apresentou idade de $20,0 \pm 1,2$ anos, altura de $1,65 \pm 0,06 \mathrm{~m}$, massa corporal de $63,7 \pm 14,7 \mathrm{~kg}$ e IMC $23,4 \pm 4,9$. Em nenhuma das variáveis analisadas houve diferença significativa nas comparações, tanto para o total de acomodações e tempo para a primeira (fig. 2), quanto para as intensidades envolvidas (fig. 3). Todas as 15 voluntárias foram analisadas e não houve dados faltantes. 
Figura 2. Variáveis relacionadas à adaptação da corrente interferencial. A. Número de acomodações tanto para a forma bi quanto tetrapolar. B. Tempo despendido até primeira adaptação
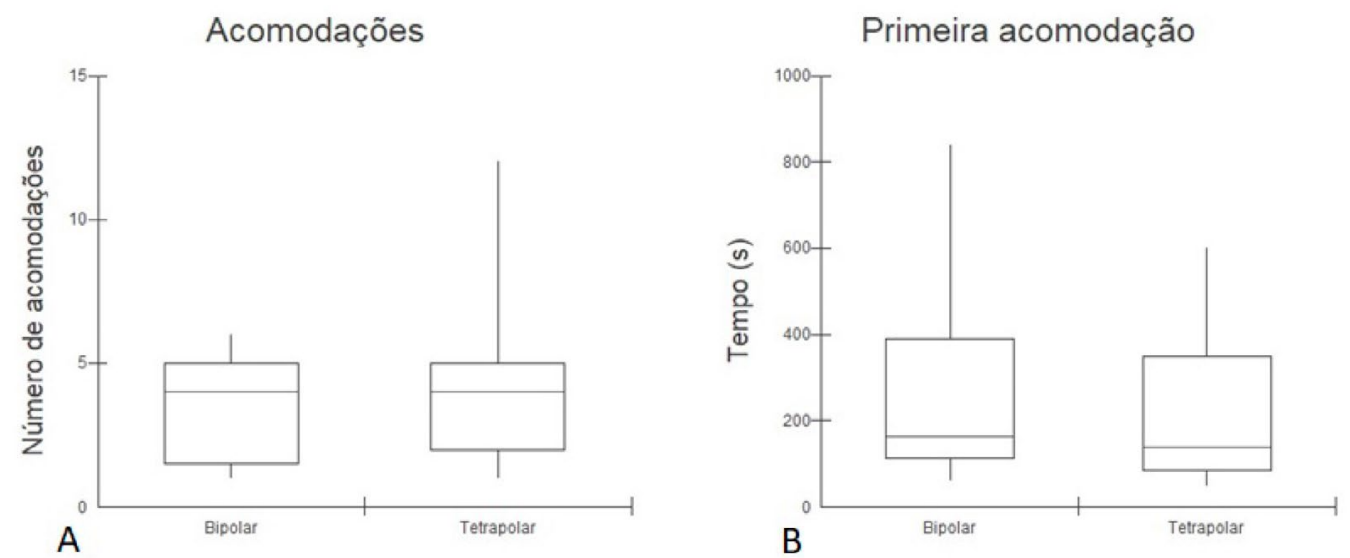

Figura 3. Variáveis relacionadas à intensidade de corrente. A. Intensidade inicial utilizada nos diferentes subgrupos. B. Elevação da intensidade quando atingida a primeira adaptação

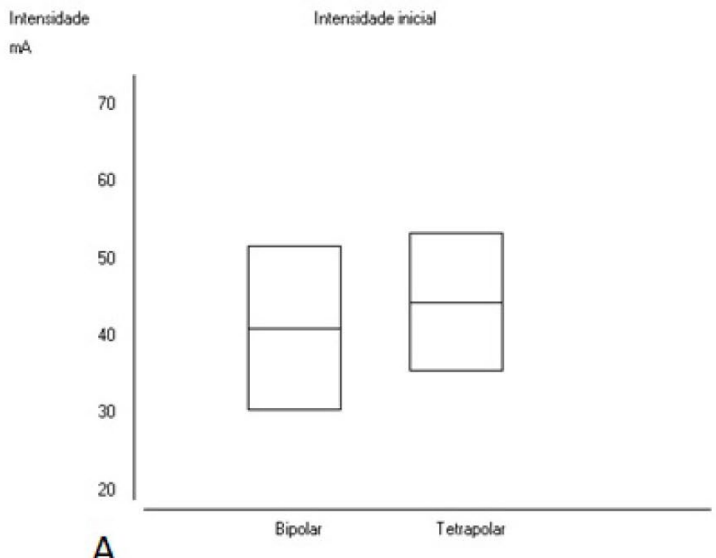

A
Elevaç5o de intensidade

Irkentida

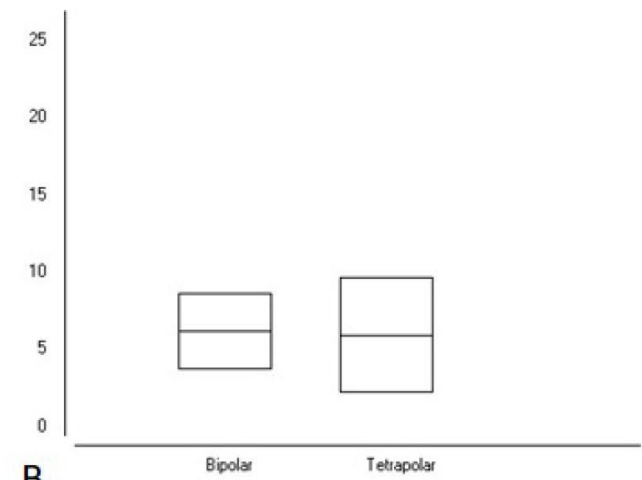

\section{Discussão}

Este estudo comparou a adaptação da corrente interferencial bipolar com a observada na forma tetrapolar em mulheres com lombalgia não específica crônica, não sendo observadas diferenças em ambos os tipos de estimulação. As duas formas de terapia são utilizadas na prática clínica e pesquisas apontam para benefícios quando utilizadas para redução de quadros álgicos $3,6,15$, sendo que a forma tetrapolar, também denominada de "verdadeira", apresenta como vantagem maior espalhamento da corrente, dando assim maior área de tratamento. $\frac{16}{}$

Em pesquisa com indivíduos saudáveis, observou-se que a forma tetrapolar apresentou maior limiar de adaptação, ou seja, demorou um maior tempo entre o início da aplicação até o momento em que o voluntário relatou necessidade de aumentar a intensidade, ao comparar com a forma bipolar. ${ }^{14}$ Contudo, em raros casos, a corrente é utilizada abaixo do limiar motor em indivíduos saudáveis. Assim, a intenção deste estudo foi testar as formas de corrente em indivíduos lombálgicos. A dor lombar não específica leva à limitação da capacidade física, o que possibilita o uso de vários recursos terapêuticos, como agentes eletrofísicos para alívio da dor, o que pode facilitar para estes pacientes a participação em um programa de exercícios.,3,17-19 Porém, a necessidade constante de readequações na intensidade da corrente pode ser dificultosa tanto para o paciente, quanto para o terapeuta, que necessita sempre estar próximo ao equipamento; desta forma, a observação de qual tipo de corrente, ou qual recurso possa garantir maior tempo sem necessidade de aumentar a intensidade devido adaptação ao estímulo mostra-se importante. 
Visto que as correntes de eletroestimulação, principalmente aquelas abaixo do limiar motor, produzem adaptação rápida, ou seja, necessitam atenção constante, algumas correntes são providas de mudanças em frequência e intensidade, com vistas a reduzir a necessidade de elevação da amplitude de corrente, como o TENS, que caracteriza-se por ser de baixa frequência, e em equipamentos atuais dispor da modalidade VIF, em que se alteram tanto duração de fase quanto frequência, mudando também a carga elétrica aplicada. Ao comparar esta modalidade com o TENS convencional e o burst, em indivíduos com dor lombar não específica, Costa et al. ${ }^{20}$ observaram que na primeira houve menor número de acomodações, apesar de não ocorrer diferenças nos limiares de adaptação. Já Silva et al._11 não observaram diferenças na adaptação ao comparar as frequências de 7, 100 e $255 \mathrm{~Hz}$ do TENS.

Para a corrente interferencial, em indivíduos saudáveis, não se observou diferenças ao analisar variações na frequência base $\frac{13}{}$, nem o uso de recursos como o $\Delta \mathrm{f}^{11}$ e diferentes rampas de apresentação ${ }^{12}$; sendo que no presente estudo também não foram observadas variações significativas entre os grupos tanto no limiar de adaptação quanto no número de adaptações, ou seja, o tempo necessário para que ocorresse a adaptação foi semelhante para os grupos, bem como a quantidade de vezes em que houve necessidade de aumentar a corrente para que o estímulo analgésico prosseguisse atuante. Ainda em voluntários saudáveis, Dallacorte et al. $\underline{22}$ compararam variações entre sexos e observaram que os homens precisaram de maiores intensidades de corrente para obter níveis agradáveis de estimulação, tendo maior limiar e menor número de acomodações. Neste estudo, mesmo tendo como desenho metodológico o cruzamento, optou-se por utilizar apenas mulheres jovens sintomáticas, e, visando reduzir uma possível influência da intensidade de corrente inicial nas acomodações, decidiu-se comparar tanto esta variável, quanto a amplitude utilizada para aumentar no primeiro episódio de adaptação, sendo que em ambas as variáveis novamente não houve diferenças estatísticas para as duas maneiras de aplicação, indicando proximidade de limiares de estimulação para ambas as formas.
Salienta-se como limitação a aplicação de uma única intervenção, neste modelo de estudo cruzado, o que pode interferir com um possível "aprendizado" da sensação de estimulação. Sugere-se que futuros estudos avaliem se a somatória de terapias pode produzir alguma influência nas variáveis em análise. Também se destaca como limitação os parâmetros e a amostra utilizada, que não reflete todos os indivíduos em que se utiliza esta forma de terapia, porém acredita-se que possa nortear o que se esperar também com indivíduos de sexo e idades diferentes.

\section{Conclusão}

Conclui-se que quando comparadas às formas bipolar e tetrapolar, não houve diferenças significativas na adaptação em mulheres jovens com lombalgia crônica não específica.

\section{Contribuições dos autores}

Lima KL, Krampe PT, Battisti WH participaram da concepção, delineamento, coleta de dados e redação do artigo científico. Artioli DP participou da concepção, delineamento, interpretação dos dados e revisão crítica do manuscrito. Bertolini GRF participou da concepção, delineamento, análise estatística dos dados da pesquisa, interpretação dos resultados e revisão crítica do artigo científico.

\section{Conflitos de interesses}

Nenhum conflito financeiro, legal ou político envolvendo terceiros (governo, empresas e fundações privadas, etc.) foi declarado para nenhum aspecto do trabalho submetido (incluindo, mas não se limitando a subvenções e financiamentos, participação em conselho consultivo, desenho de estudo, preparação de manuscrito, análise estatística, etc.).

\section{Referências}

\begin{abstract}
1. Maher C, Underwood M, Buchbinder R. Non-specific low back pain. Lancet. 2017;389(10070):736-47. https://doi.org/10.1016/ s0140-6736(16)30970-9
\end{abstract}

2. Chenot JF, Greitemann B, Kladny B, Petzke F, Pfingsten M, Schorr SG. Clinical practice guideline: Non-specific low back pain. Dtsch Arztebl Int. 2017;114(51-52):883-90. https://doi.org/10.3238/ arztebl.2017.0883 
3. Albornoz-Cabello M, Maya-Martín J, Domínguez-Maldonado G, Espejo-Antúnez L, Heredia-Rizo AM. Effect of interferential current therapy on pain perception and disability level in subjects with chronic low back pain: A randomized controlled trial. Clin Rehabil. 2017;31(2):242-9. https://doi.org/10.1177/0269215516639653

4. Franco KM, Franco YS, Oliveira NB, Miyamoto GC, Santos $\mathrm{MO}$, Liebano RE, et al. Is interferential current before pilates exercises more effective than placebo in patients with chronic nonspecific low back pain?: A randomized controlled trial. Arch Phys Med Rehabil. 2017;98(2):320-8. https://doi.org/10.1016/j. apmr.2016.08.485

5. Corrêa JB, Costa LOP, Oliveira NTB, Lima WP, Sluka KA, Liebano $R E$. Effects of the carrier frequency of interferential current on pain modulation and central hypersensitivity in people with chronic nonspecific low back pain: A randomized placebocontrolled trial. Eur J Pain. 2016;20(10):1653-66. https://doi. org/10.1002/ejp.889

6. Serafim A, Rabel J, Neves M, Silva T, Bertolini G. Evaluation of acute low back pain in women after treatment with interferential current. J Pre-Clinical Clin Res. 2019;13(2):57-60. https://doi. org/10.26444/jpccr/109752

7. Franco YR, Franco KF, Silva LA, Silva MO, Rodrigues MN, Liebano $\mathrm{RE}$, et al. Does the use of interferential current prior to pilates exercises accelerate improvement of chronic nonspecific low back pain? Pain Manag. 2018;8(6):465-74. https://doi.org/10.2217/pmt2018-0034

8. Fuentes JP, Olivo SA, Magee DJ, Gross DP. Effectiveness of interferential current therapy in the management of musculoskeletal pain: a systematic review and meta-analysis. Phys Ther. 2010;90(9):1219-38. https://doi.org/10.2522/ptj.20090335

9. Goats GC. Interferential current therapy. Br J Sport Med. 1990;24(2):87-92. https://doi.org/10.1136/bjsm.24.2.87

10. Krueger-Beck E, Nogueira-Neto GN, Neves EB, Nohama P. Potencial de ação: do estímulo à adaptação neural. Fisioter mov. 2011;24(3):535-47. https://doi.org/10.1590/50103$\underline{51502011000300018}$

11. Pivetta KM, Bertolini GRF. Efeitos do $\Delta \mathrm{F}$ sobre a acomodação da corrente interferencial em sujeitos saudáveis. Rev Bras Med Esporte. 2012;18(5):330-2. https://doi.org/10.1590/S151786922012000500009

12. Guerra TEC, Bertolini GRF. Efeitos da variação da rampa de entrega do $\Delta \mathrm{F}$ sobre a acomodação da corrente interferencial em mulheres saudáveis. Rev Dor. 2012;13(1):25-9. https://doi. org/10.1590/S1806-00132012000100005

13. Grando F, Bernardino G, Carvalho J, Bosco J, Arago F, Bertolini G. Comparison of interferential current accommodation using three different base frequencies. Int J Ther Rehabil Res [Internet]. 2014;3(2):5. Disponível em: https://www.researchgate.net/ profile/Gladson-Bertolini/publication/273716261_Comparison of interferential current accommodation using three different_base_frequencies/links/55195b170cf273292e714fe1/ Comparison-of-interferential-current-accommodation-usingthree-different-base-frequencies.pdf

14. Fiori A, Cescon CLC, Galesky JDF, Santos TACD, Brancalhaõ RMC, Bertolini GRF. Comparison between bipolar and tetrapolar of the interferential current in nociceptive threshold, accommodation and pleasantness in healthy individuals. Eur J Physiother. 2014;16(4):201-5. https://doi.org/10.3109/21679169.2 014.933875

15. Okuyama EB, Yoshida GSO, Weirich RV, Assai TM, Artioli DP, Bertolini GRF. Effect of tetrapolar interferential current on primary dysmenorrhea associated with low back pain: randomized clinical trial. J Heal Sci. 2019;21(3):204. https://doi.org/10.17921/24478938.2019v21n3p204-207

16. Beatti A, Rayner A, Chipchase L, Souvlis T. Penetration and spread of interferential current in cutaneous, subcutaneous and muscle tissues. Physiotherapy. 2011;97(4):319-26. https://doi. org/10.1016/j.physio.2011.01.008

17. Urrútia G, Burton AK, Morral A, Bonfill X, Zanoli G. Neuroreflexotherapy for non-specific low-back pain. Cochrane Database Syst Rev. 2004;(2):CD003009. Citado em: PMID: 15106186

18. Goubert D, Van Oosterwijck J, Meeus M, Danneels L. Structural changes of lumbar muscles in non-specific low back pain. Pain Physician. 2016;19(7):E985-E1000. Citado em: PMID: 27676689

19. Karvat J, Antunes JS, Bertolini GRF. Corrente interferencial como forma de tratamento em pacientes com dor lombar. Publ UEPG Ciencias Biol e da Saude [Internet]. 2016;22(1):7-13. https:// revistas2.uepg.br/index.php/biologica/article/view/6694

20. Costa ER, Roth F, Pauli G, Gozzblr VB, Anguera MG, Bertolini GRF. Accommodation and pleasantness of different forms of transcutaneous electrical nerve stimulation in individuals with nonspecific lumbar pain. Int Phys Med Rehabil J. 2018;4(2):86-9. https://doi.org/10.15406/ipmrj.2019.04.00179

21. Silva DO, Ferreira AS, Gonçalves AV, Costa MD, Zilio M, Fréz $A R$, et al. Efeitos de diferentes frequências da estimulação elétrica nervosa transcutănea em relação à acomodação e à agradabilidade. Sci Med. 2014;24(3):264-8. https://doi. org/10.15448/1980-6108.2014.3.17739

22. Dallacorte DA, Sprizon GS, Bueno KS, Hotz PG, Aragão FA, Bertolini GRF. Comparison of the effects of interferential current between male and female healthy adults. Sci Med. 2017;27(3):ID27660. https://doi.org/10.15448/1980$\underline{6108.2017 .3 .27660}$ 